\title{
Inflammatory cytokine levels in synovial fluid 3,4 days postoperatively and its correlation with early-phase functional recovery after anterior cruciate ligament reconstruction: a cohort study
}

\author{
Makiko Inoue ${ }^{1,2}$, Takeshi Muneta ${ }^{2}$, Miyoko Ojima², Kaori Nakamura² ${ }^{2}$ Hideyuki Koga ${ }^{2}$, Ichiro Sekiya ${ }^{3}$, \\ Mutsumi Okazaki ${ }^{1}$ and Kunikazu Tsuji ${ }^{4^{*}}$
}

\begin{abstract}
Background: Synovial fluid was collected prior to and at 3 to 4 days after ACL reconstruction to investigate the correlation between inflammatory cytokine levels in the acute phase after surgery and physical functional recovery at 3 months postoperatively.

Methods: For this purpose, 79 patients with ACL reconstruction using semitendinosus tendons were included in the study. Median days from injury to surgery were 80 days (13-291 days). Synovial fluid was obtained just before surgery and at 3 to 4 days after surgery. Physical activity of each patient was evaluated at 3 months postoperatively, and scored from 0 (hard to walk) to 5 (run). Patients able to jog (score 4) or run (score 5) were considered as the "quick recovery" group and others (scores 1-3) as the "delayed recovery" group.

Results: Physical activity recovery scores in the early surgery group (preoperative period less than 60 days; Group I) were significantly better than those in the delayed surgery group (Group II). Among the cytokines tested, TNF-alpha and IL10 levels in synovial fluid were significantly higher in Group II at 3 to 4 days postoperatively, while levels of these cytokines were quite comparable preoperatively between the groups. Increased IL1-beta expression was noted in the delayed recovery group at 3 to 4 days postoperatively. In addition, levels of IL6, IL10 and IFN-gamma also tended to increase in patients with delayed recovery.
\end{abstract}

Conclusion: Delayed ACL reconstruction increases levels of inflammatory cytokines in synovial fluid after surgery and correlates with a prolonged recovery of short-period physical activity of the patients.

Keywords: Anterior cruciate ligament, Synovial fluid, Inflammatory cytokines, Recovery after surgery, Preoperative period

\section{Background}

The anterior cruciate ligament (ACL) graft undergoes a long-term healing process known as "ligamentization" (Claes et al. 2011; Janssen \& Scheffler 2014; Scheffler et al. 2008). It will be important for surgeons to classify

\footnotetext{
* Correspondence: tsuji.orj@tmd.ac.jp

${ }^{4}$ Department of Cartilage Regeneration, Graduate School of Medical Sciences, Tokyo Medical and Dental University, 1-5-45 Yushima, Bunkyo-ku, Tokyo

113-8510, Japan

Full list of author information is available at the end of the article
}

the variation of the healing process after surgery in individual patients.

A review article regarding the return to sports after ACL reconstruction revealed that on average, $81 \%$ of patients returned to any sport, $65 \%$ returned to their pre-injury level of sport, and $55 \%$ returned to a competitive level sport after surgery (Ardern et al. 2014; Petersen et al. 2014). Age, preoperative period, inflammatory reaction and pain are considered to affect the functional recovery after ACL reconstruction from the early phase (Czuppon et al. 2014; 
Feller \& Webster 2013; Petersen et al. 2014). A systematic review classified the injury-to-surgery interval as either early (ranging from within 2 days to 7 months) or delayed (ranging from 3 weeks to 24 years) and found that 8 articles recommended early reconstruction, whereas the majority of the literature found no difference in outcome between early and delayed surgery (Andernord et al. 2013). These indicate that the effect of the timing of surgery after injury is still controversy (Krutsch et al. 2015; Kwok et al. 2013).

"Synovial fluid has been extensively investigated for joint degeneration-related markers upon ACL injury and after ACL reconstruction surgery" (Åhlén et al. 2015; Harkey et al. 2015; Mendias et al. 2013). Bigoni, et al. reported increased levels of pro-inflammatory cytokines (IL-6 and IL-8) in the acute phase of inflammation following ACL injury (Bigoni et al. 2013). Our previous study suggested that osteopontin levels in synovial fluid are associated with the severity of joint pain and cartilage degradation after ACL rupture (Yamaga et al. 2012).

The purpose of this study was to measure inflammatory cytokines in synovial fluid preoperatively and at 3 to 4 days after ACL reconstruction surgery and to investigate the correlation between each cytokine and the functional recovery status at 3 months postoperatively. The hypothesis of this study was that the levels of inflammatory cytokines in synovial fluid are inversely correlated with the functional recovery of the patient at 3 months after surgery.

\section{Methods}

\section{Patients}

This study was approved by the Ethics Committee of Tokyo Medical and Dental University. The approval number was 455. All patients included in this study gave their full written, informed consent for participation in this study prior to the ACL reconstruction surgery. Synovial fluid was obtained just before surgery and at 3 to 4 days after surgery.

Seventy-nine patients who underwent ACL reconstruction from January 2013 through April 2014 were included in this study (female: 40, male: $39 ; 12-62$ yearold, median 19 year-old; average Tegner score $7.0+/-0.9$, Table 1). Median days from injury until index surgery were 80 days (13-291 days). Ninety-six percent (76 out of 79 ) of patients did not have any knee injury on the contralateral side. Patients with a preoperative period of less than 60 days were defined as the early surgery group (Group I), while patients with a preoperative period 60 days and longer were defined as the delayed surgery group (Group II). All surgeries and follow-ups were conducted by surgeons of the knee and sports medicine group in our university hospital.

\section{Surgical technique}

An anatomic double-bundle technique using two autologous double-stranded semitendinosus tendons was employed for a remnant-preserving ACL reconstruction by the behind-remnant approach (Muneta et al. 2015). Tibial tunnels were created in the original remnant and just lateral to the medial tibial eminence. Fixation was achieved by pull-out technique using two EndoButtons CL BTB (Smith \& Nephew, Andover, FL), and two anchor staples (Meira corporation, Aichi, Japan). Three \#2 strong sutures were used for tibial fixation. The initial tension was set proportionally to the graft diameter at $20^{\circ}$ flexion (Koga et al. 2015).

\section{Postoperative management}

The patients followed a rehabilitation protocol described before (Muneta et al. 2015). The patients were encouraged to initiate knee range motion from the $3^{\text {rd }}$ day postoperatively. Patients were allowed to bear weight using an immobilized brace (Knee Brace ${ }^{\circ}$, Alcare, Tokyo, Japan) from the $2^{\text {nd }}$ day postoperatively as tolerated. Usually, braces and crutches were no longer used after 4 weeks postoperatively. Jogging was allowed at 2.5 months post-surgery when a single-leg half squat was achieved safely on the injured leg. The patients were encouraged to practice specialized training for their intended athletic activities after $80 \%$ of their full running capacity was achieved. The patients were expected to return to their pre-injury athletic activities 6 months or more after surgery based on individual recovery of athletic performance.

\section{Scoring of physical activity at 3 months after ACL reconstruction}

Physical activity of each patient at 3 months after surgery was evaluated and scored according to the criteria indicated in Table 2. Patients who can jog (score 4) or run (score 5) were classified as the "quick recovery" group, and others (scores 1, 2, or 3) were classified as the "delayed recovery" group. Jogging was allowed after 2.5 months postoperatively if the patient could perform single-leg squatting easily on each limb.

\section{Cytokine quantitation}

Cytokine levels in synovial fluid were quantitated by multiplex ELISA system (V-PLEX human proinflammatory panel, Meso Scale Discovery, MD). Levels of TNF-alpha, IL1-beta, IL2, IL6, IL8, IL10, and IFNgamma in synovial fluid were quantitated according to the manufacturer's protocol. 
Table 1 Patient information and clinical outcomes

\begin{tabular}{|c|c|c|c|c|}
\hline & Total & $\begin{array}{l}\text { Group I (Preoperative } \\
\text { periods }<60 \text { days) }\end{array}$ & $\begin{array}{l}\text { Group II (Preoperative } \\
\text { periods } \geq 60 \text { days) }\end{array}$ & $P$ value \\
\hline Number of patients & 79 & 27 & 52 & \\
\hline Periods from injury until index surgery (day) & $80(13-291)$ & $44(13-58)$ & $119(60-291)$ & \\
\hline \multicolumn{5}{|l|}{ Median (range) } \\
\hline Age (year) & $19(12-62)$ & $21(14-52)$ & $19(12-62)$ & 0.84 \\
\hline \multicolumn{5}{|l|}{ Median (range) } \\
\hline Gender $(M ; F)$ & $39 ; 40$ & $15 ; 12$ & $25 ; 27$ & 0.63 \\
\hline Tegner score & $7.0 \pm 0.9$ & $7.3 \pm 0.8$ & $6.9 \pm 0.9$ & 0.10 \\
\hline \multicolumn{5}{|l|}{ Average $\pm \mathrm{SD}$} \\
\hline Meniscus injury & $28(35.4 \%)$ & $10(37.0 \%)$ & $18(34.6 \%)$ & 1 \\
\hline Recovery score & $3.2 \pm 0.8$ & $3.4 \pm 0.7$ & $3.1 \pm 0.8$ & 0.04 \\
\hline Average \pm SD & & & & \\
\hline
\end{tabular}

\section{Statistical analysis}

EZR was employed for statistical analyses (Kanda 2013). Student's $t$-test, Mann-Whitney's $U$-test, Fisher's exact test, and the Jonckheere- Terpstra trend test were employed for the statistical analyses in this study. Prior to statistical analyses, the Smirnov-Grubbs test was performed to exclude outliers. $P$ values less than 0.05 were considered as significant.

\section{Results}

\section{Early and delayed surgery groups}

According to the 5-grade of physical activity score, the score of Group I was significantly better than that of Group II (Fig. 1). No significant differences in age (Student's $t$-test), gender (Fisher's exact test), Tegner score (Mann-Whitney's $U$ test), and injury history (Fisher's exact test) were observed between the groups (Table 1).

\section{Cytokine levels in synovial fluid preoperative and at 3 to}

\section{4 days after surgery}

Prior to ACL reconstruction, it was impossible to obtain synovial fluid by arthrocentasis in half of the patients because of the small volume accumulation in the joint. In all patients, synovial fluid volume surged 3 to 4 postoperatively (average $27+/-12.5 \mathrm{ml}$ ).

Table 2 Physical activity at 3 months post surgery

\begin{tabular}{lll}
\hline Score & Activity & Category \\
\hline 1 & Hard to walk & Delayed recovery \\
2 & Walking & \\
3 & Stair climbing & \\
4 & Jogging & Quick recovery \\
5 & Running & \\
\hline
\end{tabular}

Multiplex ELISA indicated that the levels of TNF alpha, IL1 beta, IL2, IL6, IL8, IL10, and IFN gamma were significantly increased at 3 to 4 days after surgery in comparison to preoperative levels, although the degree of the increased expression level of each cytokine varied in each patient (Fig. 2).

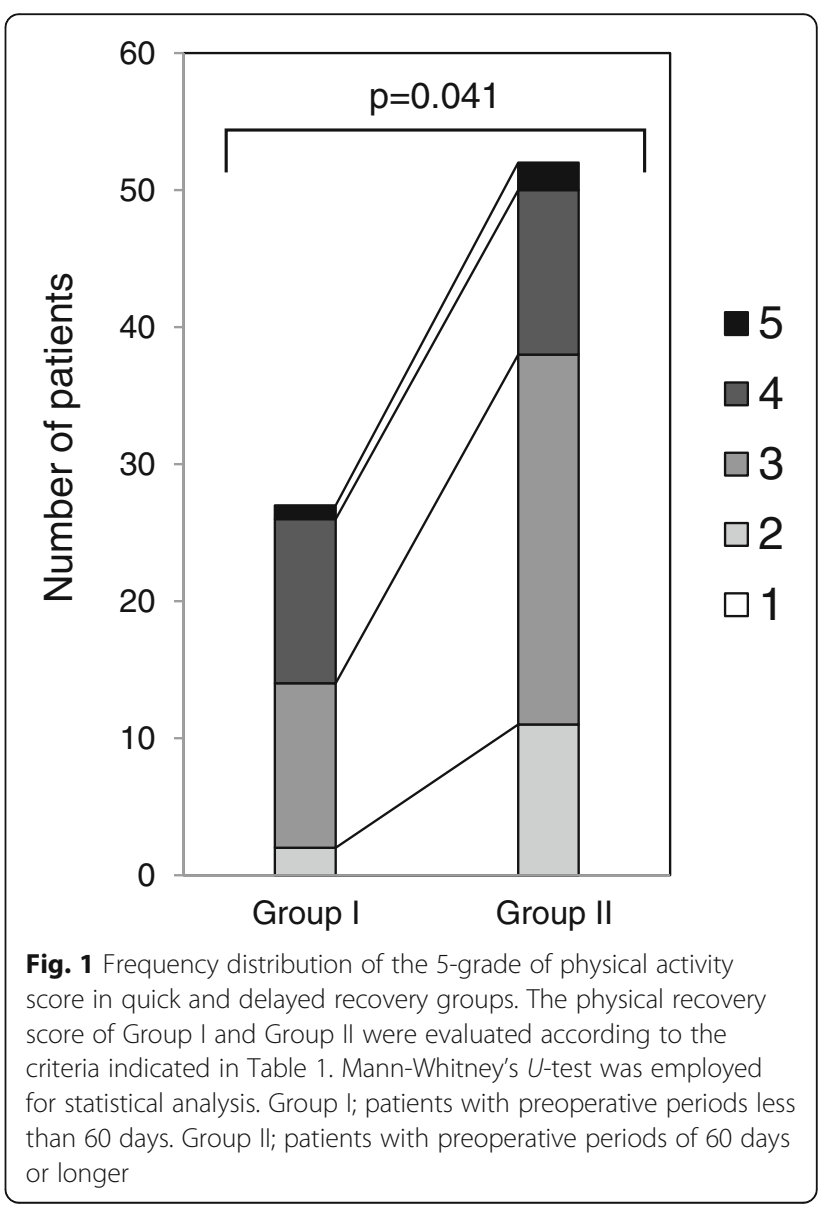



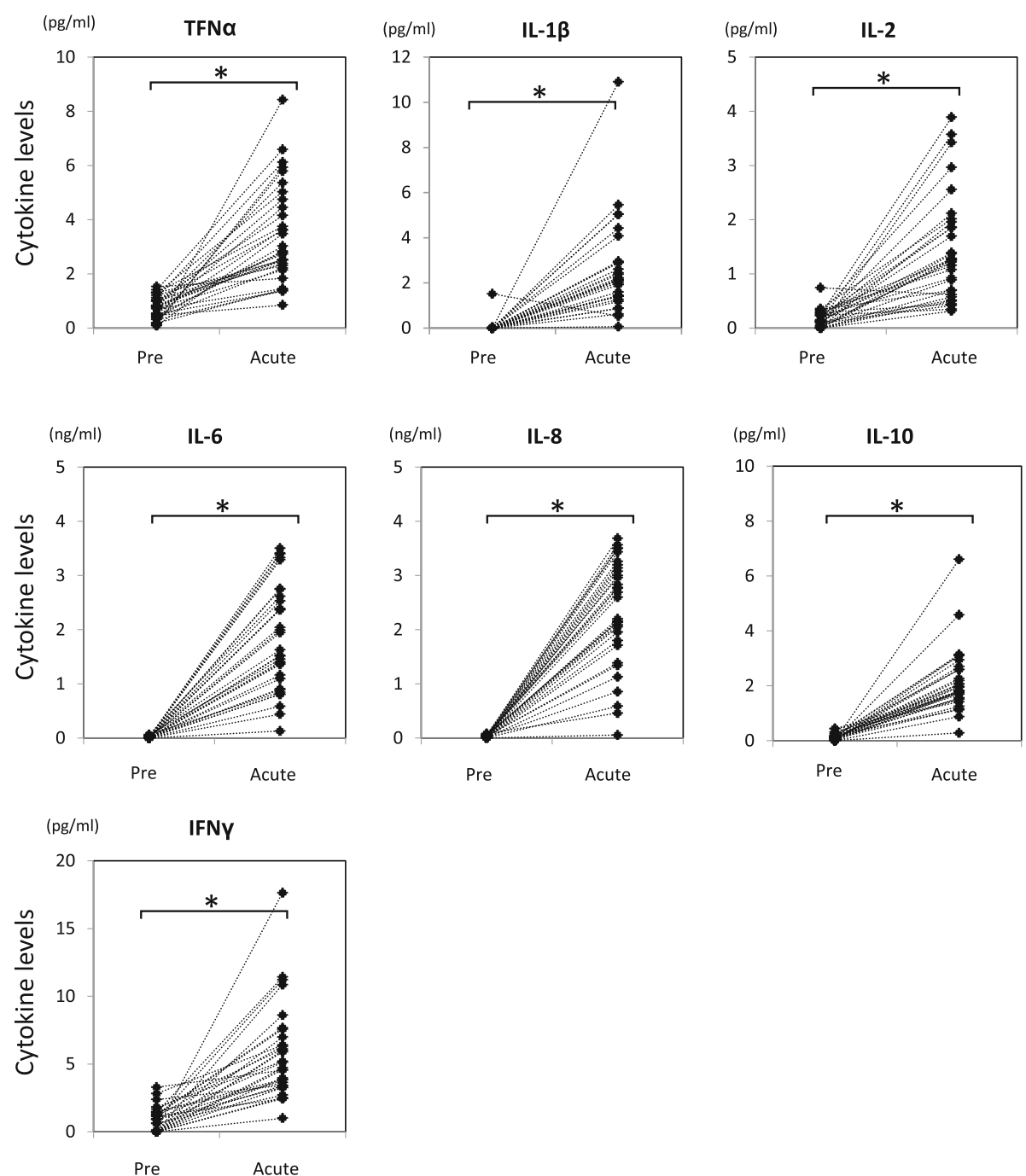

Fig. 2 Levels of cytokines in synovial fluid were significantly increased after index ACL reconstructive surgery. Cytokine levels in synovial fluid were quantitated by Multiplex ELISA. Student's $t$-test was employed for statistical analysis (Asterisk indicates $p$-value less than 0.05 ). Pre; synovial fluid harvested at surgery. Acute; synovial fluid harvested at 3 to 4 days after surgery

Levels of TNF alpha and IL10 in synovial fluid at 3 to 4 days after surgery were significantly increased in delayed surgery group

Among the cytokines tested, TNF alpha and IL10 levels in synovial fluid were significantly higher in Group II (Fig. 3), while the levels of these cytokines prior to index surgery were quite comparable among the patients in both Group I and Group II (Fig. 4).

Physical activity recovery at 3 months and inflammatory cytokines at 3 to 4 days postoperatively

To examine if elevated levels of cytokines in synovial fluid in the acute phase after ACL reconstruction affected recovery status at 3 months post-surgery, acute phase cytokine levels were compared to patients' 5-grade of physical activity scores at 3 months
(Table 2). As indicated in Fig. 5, levels of IL1 beta significantly increased in the delayed recovery group (score 1-3) when compared to the quick recovery group (score 4-5) based on the patients' physical activity at 3 months $(p=0.03)$. In addition, levels of IL6, IL10 and IFN gamma were also increased in patients with lower physical activity at 3 months $(p=$ 0.08 , respectively).

\section{Discussion}

The most important findings of this study was that the severity of joint inflammation in the acute phase after surgery was significantly higher if the timing of index ACL reconstruction surgery was delayed. The physical activity recovery was greatly affected by the severity of acute joint inflammation in the early phase after surgery. 

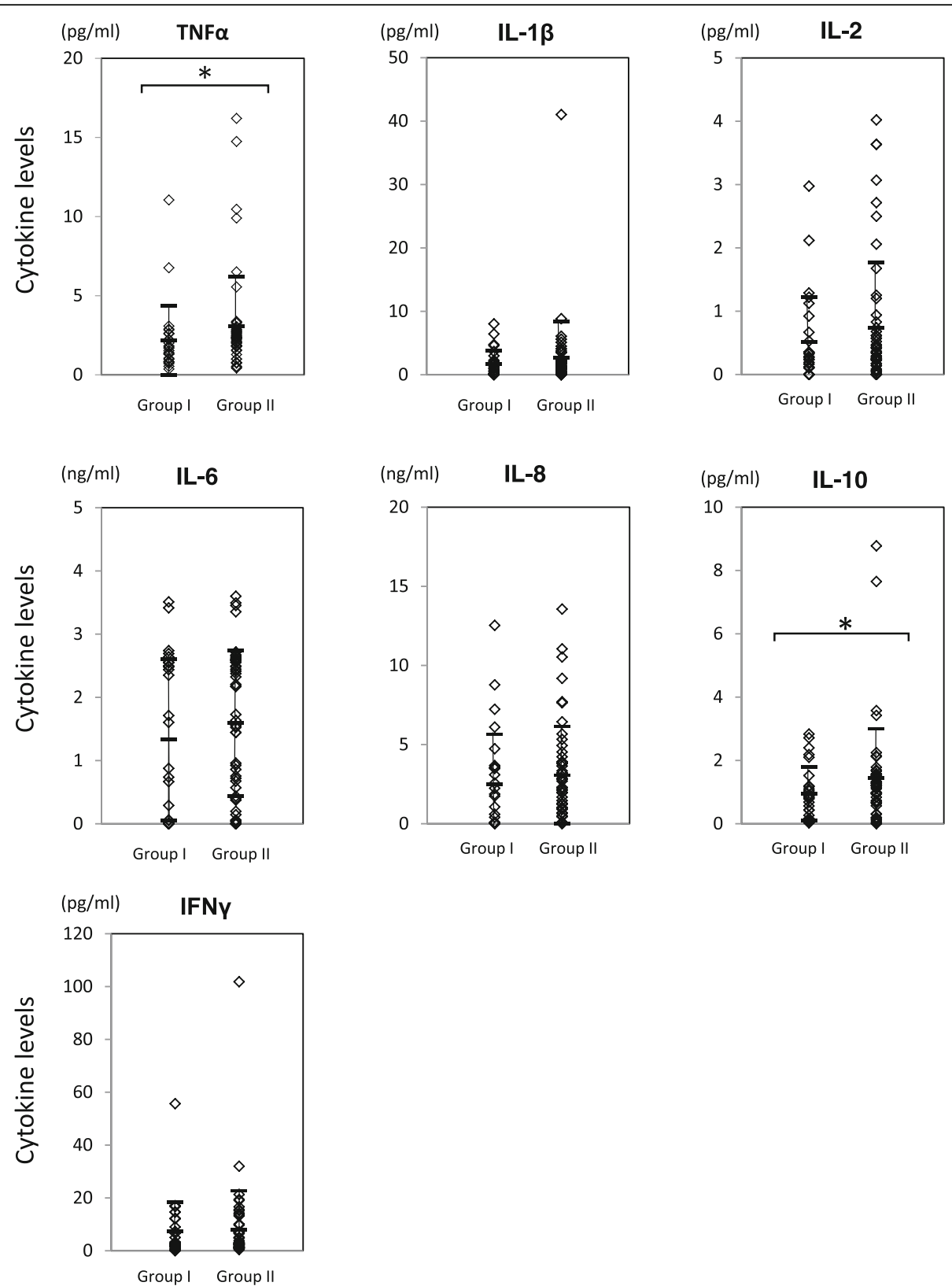

Fig. 3 Levels of cytokines in synovial fluid at 3 to 4 days post ACL reconstruction surgery. Cytokine levels in synovial fluid at 3 to 4 days post ACL reconstruction surgery were quantitated by Multiplex ELISA. The Mann-Whitney's U-test was employed for statistical analysis (Asterisk indicates $p$-value less than 0.05). Group l; patients with preoperative periods less than 60 days. Group Il; patients with preoperative periods of 60 days or longer

Previous studies have revealed several factors that influence the prognosis of ACL reconstruction surgery (Andernord et al. 2013; Czuppon et al. 2014; Feller \& Webster 2013; Petersen et al. 2014). Among these factors, timing of surgical treatment after ACL injury is still controversial (Krutsch et al. 2015; Kwok et al. 2013). In this study, we divided patients into two groups according to the preoperative periods of 60 days. Patients are assumed to be able to walk easily and navigate up/down stairs with or without difficulty by 60 days after injury on our rehabilitation protocol after ACL reconstruction. Therefore, 60 days may represent a reasonable time point to determine the indication of an acute ACL surgery after injury (Andernord et al. 2013). In this study, we focused on the short period physical recovery because the early recovery status significantly affects the recovery to return to sports in the long run. The results showed that the physical activity at 3 months after ACL reconstruction in Group II was significantly lower than that in Group I. A systematic review reported that 

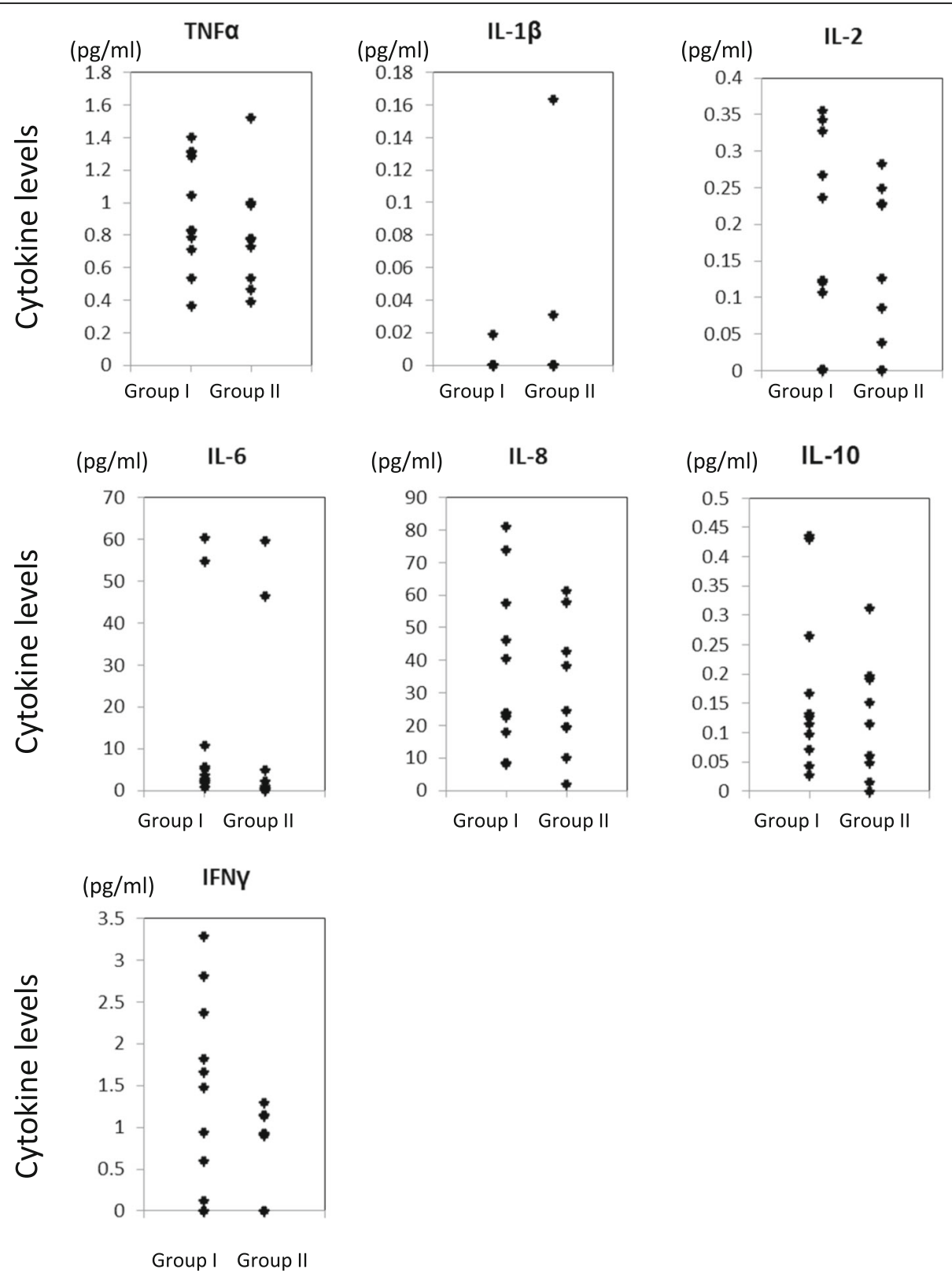

Fig. 4 Levels of cytokines in synovial fluid before ACL reconstruction surgery. Cytokine levels in synovial fluid before ACL reconstruction surgery were quantitated by Multiplex ELISA. The Mann-Whitney's U-test was employed for statistical analysis (Asterisk indicates $p$-value less than 0.05). Group I; patients with preoperative periods less than 60 days $(n=13)$. Group II; patients with preoperative periods of 60 days or longer $(n=11$

among the studies searched (49 studies in total), $39 \%$ of studies permitted running at 3 months (Harris et al. 2014). The results of the current study would provide a basic biologic background for the review. Andernord et al. (Andernord et al. 2013) showed patients that underwent reconstruction at a sub-acute stages (2-12 weeks) after ACL injury had higher activity levels after 2-2.5 years than those who underwent delayed surgery by 12-24 months. We need to follow up the patients of this study to find out the relationship of the cytokine levels in joint fluid 3,4 days postoperatively and the long-term recovery after ACL reconstruction between the Group I and Group II.

TNF alpha was significantly increased in the patients of Group II (Fig. 3). Patients with a delayed recovery rate at 3 months after surgery had higher levels of the inflammatory cytokines IL1 beta, IL6, and IFN gamma at 3 to 4 days after surgery (Fig. 5) although it is unclear why cytokine levels were increased in patients with a longer preoperative period (Group II). In addition to the proinflammatory cytokines (TNF alpha, IL1 beta, IL6 and IFN gamma), levels of an anti-inflammatory cytokine, 


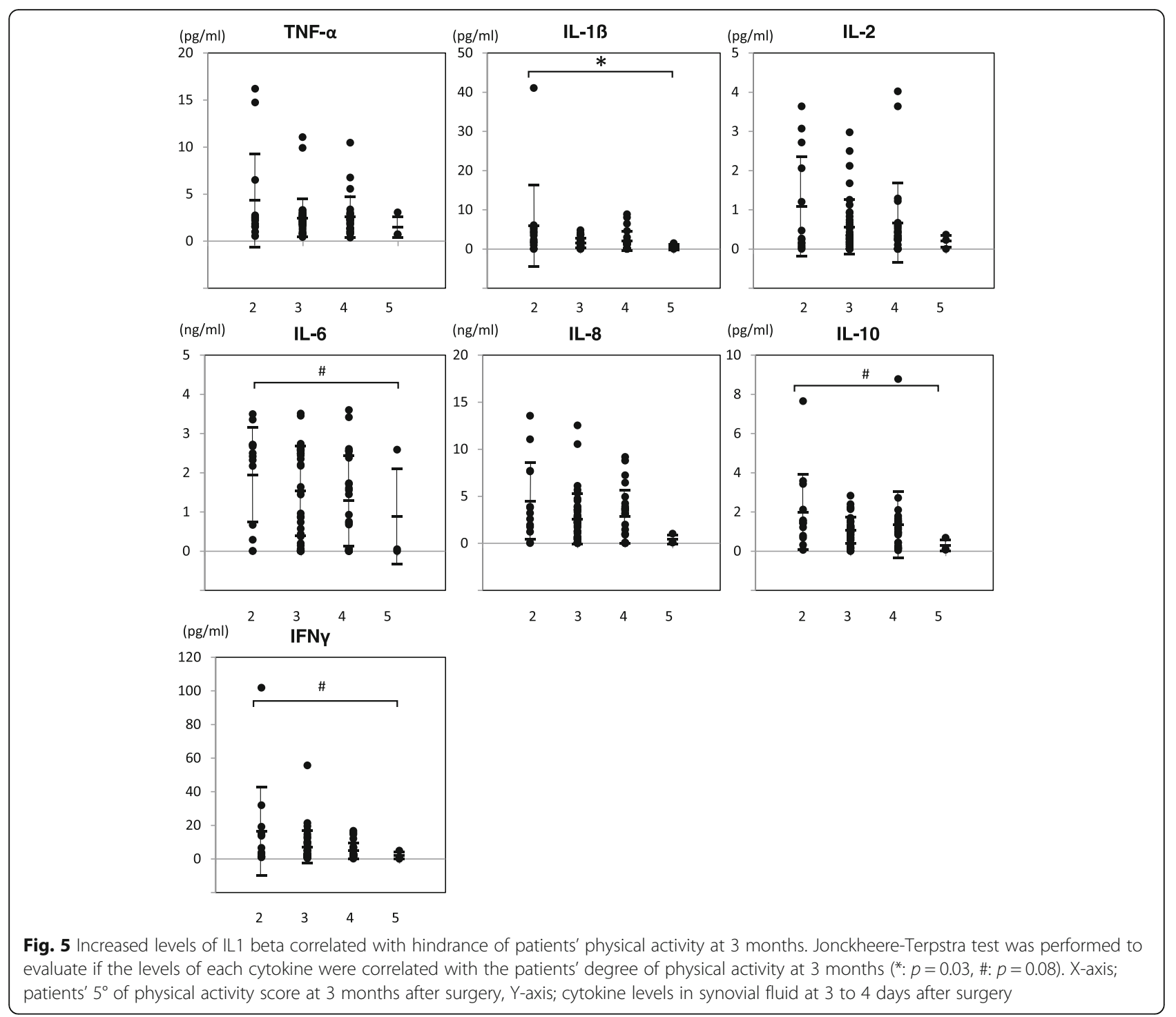

IL10, in the synovial fluid at 3 to 4 days after surgery were also significantly increased in the patients of Group II (Fig. 3) and in patients with delayed physical activity recovery at 3 months (Fig. 5) (Schulze-Tanzil et al. 2009). John et al. and other groups indicated an increase in not only the pro-inflammatory cytokines such as TNF alpha, IL1 beta, and IL6 but also the immunoregulatory cytokine IL10 after tendon injury, although the role of IL10 on tenocytes remained unclear (John et al. 2010). The results of this study regarding IL-10 showed similar trend. IL10 is an immuno-regulatory cytokine that can downregulate active immune responses and acts mainly on $\mathrm{T}$ cells or macrophages to attenuate inflammatory cytokine production and antigen presentation. IL10 deficient mice develop spontaneous colitis, suggesting the important roles of IL10 in regulating overactive inflammatory responses (Gomes-Santos et al. 2012). Physiological functions of
IL10 during the healing process after ACL reconstruction surgery has yet to be elucidated, however, our data suggest that the appropriate inflammatory response greatly affect the recovery process after surgery.

A limitation of the current study was that it was performed retrospectively without reasonable control. Also, it was not investigated whether the short-term outcome affects the longer-term clinical outcome of the patients of the study. Future studies will include the investigation of longer-term outcomes of the patients in the current study, the investigation of whether the elevated cytokines in the patients of Group II have an effect on the progress of osteoarthritic changes during longer- term follow-up, and the investigation of modifications in the early phase after surgery to improve the early phase outcome and the longer-term results after ACL reconstruction. 


\section{Conclusion}

Delayed anterior cruciate ligament reconstruction surgery increases inflammatory cytokine levels in synovial fluid after surgery and hinders short-period recovery of the patients.

\section{Abbreviations}

ACL: Anterior cruciate ligament; IFN: Interferon; IL: Interleukin; TNF: Tumor necrotic factor

\section{Acknowledgment}

We really thank Ms. Aiko Yamada and Ms. Risa Tada for their wonderful administrative support to this study.

\section{Funding}

This study was supported by the Japan Society for the Promotion of Science (JSPS). This study was also supported by "the Project for Realization of Regenerative Medicine" from the Ministry of Education, Culture, Sports, Science and Technology (MEXT; Japan), by the Health and Labor Sciences Research Grant - Research on Regenerative Medicine for Clinical Application, and by "the Research Project on Elucidation of Chronic Pain" from Japan Agency for Medical Research and Development (AMED).

\section{Availability of data and materials}

Data will be available upon request.

\section{Competing interests}

The authors declare that they have no competing interest.

\section{Authors' contributions}

Ml carried out the ELISA study and statistical analysis. MO, KN, HK, IS, and MO participated human sample collection and data interpretation. TM and KT designed this study, carried out the ELISA study, participated data interpretation, and drafted the manuscript. All authors read and approved the final manuscript.

\section{Consent for publication}

All patients included in this study gave their full written, informed consent for publication prior to the $A C L$ reconstruction surgery.

\section{Ethics approval and consent to participate}

This study was approved by the Ethics Committee of Tokyo Medical and Dental University. The approval number was 455. All patients included in this study gave their full written, informed consent for participation prior to the $\mathrm{ACL}$ reconstruction surgery.

\section{Author details}

'Department of Plastic and Reconstructive Surgery, Graduate School of Medical Sciences, Tokyo Medical and Dental University, 1-5-45 Yushima, Bunkyo-ku, Tokyo 113-8510, Japan. ²Department of Joint Surgery and Sports Medicine, Graduate School of Medical Sciences, Tokyo Medical and Dental University, 1-5-45 Yushima, Bunkyo-ku, Tokyo 113-8510, Japan. ${ }^{3}$ Center for Stem Cell and Regenerative Medicine, Tokyo Medical and Dental University, 1-5-45 Yushima, Bunkyo-ku, Tokyo 113-8510, Japan. ${ }^{4}$ Department of Cartilage Regeneration, Graduate School of Medical Sciences, Tokyo Medical and Dental University, 1-5-45 Yushima, Bunkyo-ku, Tokyo 113-8510, Japan.

Received: 22 August 2016 Accepted: 24 October 2016

Published online: 02 November 2016

\section{References}

Åhlén M, Roshani L, Lidén M, Struglics A, Rostgård-Christensen L, Kartus J (2015) Inflammatory cytokines and biomarkers of cartilage metabolism 8 years after anterior cruciate ligament reconstruction: results from operated and contralateral knees. Am J Sports Med 43:1460-1466. doi:10.1177/ 0363546515574059

Andernord D, Karlsson J, Musahl V, Bhandari M, Fu FH, Samuelsson K (2013) Timing of surgery of the anterior cruciate ligament. Arthroscopy 29 : 1863-1871. doi:10.1016/j.arthro.2013.07.270
Ardern CL, Taylor NF, Feller JA, Webster KE (2014) Fifty-five per cent return to competitive sport following anterior cruciate ligament reconstruction surgery: an updated systematic review and meta-analysis including aspects of physical functioning and contextual factors. Br J Sports Med 48:1543-1552. doi:10.1136/bjsports-2013-093398

Bigoni M, Sacerdote P, Turati M, Franchi S, Gandolla M, Gaddi D, Moretti S, Munegato D, Augusti CA, Bresciani E, Omeljaniuk RJ, Locatelli V, Torsello A (2013) Acute and late changes in intraarticular cytokine levels following anterior cruciate ligament injury. J Orthop Res 31:315-321. doi:10.1002/jor. 22208

Claes S, Verdonk P, Forsyth R, Bellemans J (2011) The "Ligamentization" Process in Anterior Cruciate Ligament Reconstruction: What Happens to the Human Graft? A Systematic Review of the Literature. Am J Sports Med 39:2476-2483. doi:10.1177/0363546511402662

Czuppon S, Racette BA, Klein SE, Harris-Hayes M (2014) Variables associated with return to sport following anterior cruciate ligament reconstruction: a systematic review. Br J Sports Med 48:356-364. doi:10.1136/bjsports-2012091786

Feller J, Webster KE (2013) Return to sport following anterior cruciate ligament reconstruction. Int Orthop 37:285-290. doi:10.1007/s00264-012-1690-7

Gomes-Santos AC, Moreira TG, Castro-Junior AB, Horta BC, Lemos L, Cruz DN, Guimarães MAF, Cara DC, McCafferty DM, Faria AMC (2012) New insights into the immunological changes in $\mathrm{IL}-10$-deficient mice during the course of spontaneous inflammation in the gut mucosa. Clin Dev Immunol. doi:10.1155/2012/560817

Harkey MS, Luc B, Golightly YM, Thomas A, Driban JB, Hackney yy A, Pietrosimone B (2015) Osteoarthritis-related biomarkers following anterior cruciate ligament injury and reconstruction: a systematic review. Osteoarthr Cartil 23:1-12. doi:10.1016/j.joca.2014.09.004

Harris JD, Abrams GD, Bach BR, Williams D, Heidloff D, Bush-Joseph CA, Verma NN, Forsythe B, Cole BJ (2014) Return to sport after ACL reconstruction. Orthopedics 37:e103-e108. doi:10.3928/01477447-20140124-10

Janssen RPA, Scheffler SU (2014) Intra-articular remodelling of hamstring tendon grafts after anterior cruciate ligament reconstruction. Knee Surg Sports Traumatol Arthrosc 22:2102-2108. doi:10.1007/s00167-013-2634-5

John T, Lodka D, Kohl B, Ertel W, Jammrath J, Conrad C, Stoll C, Busch C, Schulze-Tanzil G (2010) Effect of pro-inflammatory and immunoregulatory cytokines on human tenocytes. J Orthop Res 28:1071-1077. doi:10.1002/ jor.21079

Kanda $Y$ (2013) Investigation of the freely available easy-to-use software "EZR" for medical statistics. Bone Marrow Transplant 48:452-458. doi:10.1038/bmt.2012.244

Koga H, Muneta T, Yagishita K, Watanabe T, Mochizuki T, Horie M, Nakamura T, Otabe K, Sekiya I (2015) Evaluation of a behind-remnant approach for femoral tunnel creation in remnant-preserving double-bundle anterior cruciate ligament reconstruction - Comparison with a standard approach. Knee 22:249-255. doi:10.1016/j.knee.2015.02.017

Krutsch W, Zellner J, Baumann F, Pfeifer C, Nerlich M, Angele P (2015) Timing of anterior cruciate ligament reconstruction within the first year after trauma and its influence on treatment of cartilage and meniscus pathology. Knee Surg Sports Traumatol Arthrosc. doi:10.1007/s00167-015-3830-2

Kwok CS, Harrison T, Servant C (2013) The optimal timing for anterior cruciate ligament reconstruction with respect to the risk of postoperative stiffness. Arthroscopy 29:556-565. doi:10.1016/j.arthro.2012.09.005

Mendias CL, Lynch EB, Davis ME, Enselman ERS, Harning JA, Dewolf PD, Makki TA Bedi A, Sibilsky Enselman ER, Harning JA, Dewolf PD, Makki TA, Bedi A (2013) Changes in circulating biomarkers of muscle atrophy, inflammation, and cartilage turnover in patients undergoing anterior cruciate ligament reconstruction and rehabilitation. Am J Sports Med 41:1819-1826. doi:10. $1177 / 0363546513490651$

Muneta T, Koga H, Nakamura T, Horie M, Watanabe T, Yagishita K, Sekiya I (2015) A new behind-remnant approach for remnant-preserving double-bundle anterior cruciate ligament reconstruction compared with a standard approach. Knee Surgery, Sport Traumatol Arthrosc 23:3743-3749. doi:10.1007/s00167-014-3300-2

Petersen W, Taheri P, Forkel P, Zantop T (2014) Return to play following ACL reconstruction: a systematic review about strength deficits. Arch Orthop Trauma Surg 134:1417-1428, doi:10.1007/s00402-014-1992-x

Scheffler SU, Unterhauser FN, Weiler A (2008) Graft remodeling and ligamentization after cruciate ligament reconstruction. Knee Surgery, Sport Traumatol Arthrosc 16:834-842. doi:10.1007/s00167-008-0560-8 
Schulze-Tanzil G, Zreiqat H, Sabat R, Kohl B, Halder A, Müller RD, John T (2009) Interleukin-10 and articular cartilage: experimental therapeutical approaches in cartilage disorders. Curr Gene Ther 9:306-315. doi:10.2174/156652309788921044

Yamaga M, Tsuji K, Miyatake K, Yamada J, Abula K, Ju YJ, Sekiya I, Muneta T (2012) Osteopontin Level in Synovial Fluid Is Associated with the Severity of Joint Pain and Cartilage Degradation after Anterior Cruciate Ligament Rupture. PLoS One. doi:10.1371/journal.pone.0049014

\section{Submit your manuscript to a SpringerOpen ${ }^{\circ}$ journal and benefit from:}

- Convenient online submission

- Rigorous peer review

- Immediate publication on acceptance

- Open access: articles freely available online

- High visibility within the field

- Retaining the copyright to your article

Submit your next manuscript at $>$ springeropen.com 